\title{
Strong correlations of neutron star radii with the slopes of nuclear matter incompressibility and symmetry energy at saturation
}

\author{
N. Alam,,${ }^{1,2}$ B. K. Agrawal, ${ }^{1,2}$ M. Fortin, ${ }^{3}$ H. Pais,${ }^{4}$ C. Providência, ${ }^{4}$ Ad. R. Raduta, ${ }^{5}$ and A. Sulaksono ${ }^{6}$ \\ ${ }^{1}$ Saha Institute of Nuclear Physics, Kolkata 700064, India \\ ${ }^{2}$ Homi Bhabha National Institute, Anushakti Nagar, Mumbai - 400094, India \\ ${ }^{3}$ N. Copernicus Astronomical Center, Polish Academy of Science, Bartycka 18, 00-716 Warszawa, Poland \\ ${ }^{4}$ CFisUC, Department of Physics, University of Coimbra, 3004-516 Coimbra, Portugal \\ ${ }^{5}$ IFIN-HH, Bucharest-Magurele POB-MG6, Romania \\ ${ }^{6}$ Departemen Fisika, FMIPA, Universitas Indonesia, Depok 16424, Indonesia
}

(Received 17 June 2016; revised manuscript received 28 September 2016; published 9 November 2016)

\begin{abstract}
We examine the correlations of neutron star radii with the nuclear matter incompressibility, symmetry energy, and their slopes, which are the key parameters of the equation of state (EoS) of asymmetric nuclear matter. The neutron star radii and the EoS parameters are evaluated using a representative set of 24 Skyrme-type effective forces and 18 relativistic mean field models, and two microscopic calculations, all describing $2 M_{\odot}$ neutron stars. Unified EoSs for the inner-crust-core region have been built for all the phenomenological models, both relativistic and nonrelativistic. Our investigation shows the existence of a strong correlation of the neutron star radii with the linear combination of the slopes of the nuclear matter incompressibility and the symmetry energy coefficients at the saturation density. Such correlations are found to be almost independent of the neutron star mass in the range $0.6-1.8 M_{\odot}$. This correlation can be linked to the empirical relation existing between the star radius and the pressure at a nucleonic density between one and two times saturation density, and the dependence of the pressure on the nuclear matter incompressibility, its slope, and the symmetry energy slope. The slopes of the nuclear matter incompressibility and the symmetry energy coefficients as estimated from the finite nuclei data yield the radius of a $1.4 M_{\odot}$ neutron star in the range $11.09-12.86 \mathrm{~km}$.
\end{abstract}

DOI: 10.1103/PhysRevC.94.052801

The bulk properties of neutron stars are mainly governed by the behavior of the equation of state $(\mathrm{EoS})$ of highly asymmetric dense matter. The correlations of the various EoS parameters of asymmetric nuclear matter with the different properties of a neutron star, such as the crust-core transition density and pressure, radii, maximum mass, and cooling rate, have been studied [1-15]. The crust-core transition density is strongly correlated with the slope of the symmetry energy, $L_{0}$, at saturation density $\left(\rho_{0} \sim 0.16 \mathrm{fm}^{-3}\right)[5,6,11]$. However, the transition pressure is found to be strongly correlated with a linear combination of the slope and curvature of the symmetry energy at the subsaturation density $(\rho=$ $\left.0.1 \mathrm{fm}^{-3}\right)[7,11,12]$. The simultaneous determination of mass and radius of low-mass neutron stars can better constrain the product of nuclear matter incompressibility and symmetry energy slope parameter [13].

The correlations of the neutron star radii of different masses with the EoS parameters have been investigated extensively. The covariance analysis, based on a single model, suggests the existence of strong correlations of the radii of low-mass neutron stars $\left(M_{\mathrm{NS}} \sim 0.6-1.2 M_{\odot}\right)$ with the symmetry energy slope parameter $L_{0}$ [10], the correlations becoming weaker with the increase of the neutron star mass. A similar analysis for the correlations of the radii with the symmetry energy slope over a wider range of densities was performed for two different models, having different behaviors on the density dependence of the symmetry energy, and such correlations were found to be model dependent [12]. Recently, correlations of neutron star radii with the symmetry energy slope parameter and the nuclear matter incompressibility coefficient have been examined using a large set of unified EoSs, based on
Skyrme-type effective forces and relativistic mean field (RMF) models [14]. The dependence of correlations on the neutron star mass is qualitatively similar to those obtained within the covariance analysis, but the correlations are in general somewhat weaker due to the interference of the other EoS parameters, which were kept fixed in the latter case. Since the EoS for asymmetric nuclear matter is mainly governed by the nuclear matter incompressibility, symmetry energy, and their slopes at saturation density, the neutron star radii may be correlated with the linear combination of these EoS parameters, rather than each parameter individually, analogous to those found in the case of the correlation between the transition pressure and the linear combination of the slope and the curvature of the symmetry energy [7].

In this Rapid Communication, we examine the correlations of the neutron star radii with the key parameters governing the EoS of asymmetric nuclear matter. These EoS parameters are evaluated at the nuclear saturation density using a representative set of RMF models, a set of Skyrme-type models, one microscopic calculation using the BruecknerHartree-Fock (BHF) approach with the Argonne $V_{18}$ force supplemented with a three-body force of Urbana type [16], and a variational approach, specifically the Akmal-PandharipandeRavenhall (APR) EoS [17]. All models describe $2 M_{\odot}$ stars. We demonstrate that the neutron star radii over a wide range of masses $\left(0.6-1.8 M_{\odot}\right)$ are strongly correlated with the linear combination of the slopes of nuclear matter incompressibility and symmetry energy coefficients.

The EoS at a given density $\rho$ and asymmetry $\delta$ can be decomposed, to a good approximation, into the EoS for symmetric nuclear matter $e(\rho, 0)$ and the density dependent 
symmetry energy coefficient $S(\rho)$ as

$$
e(\rho, \delta)=e(\rho, 0)+S(\rho) \delta^{2},
$$

where $e(\rho, \delta)$ is the energy per nucleon at density $\rho=\rho_{n}+\rho_{p}$, and $\delta=\left(\rho_{n}-\rho_{p}\right) / \rho$ the asymmetry parameter, with $\rho_{n}$ and $\rho_{p}$ the neutron and proton densities, respectively.

The isoscalar part $e(\rho, 0)$ can be expanded as

$$
e(\rho, 0)=e\left(\rho_{0}\right)+\frac{K_{0}}{2}\left(\frac{\rho-\rho_{0}}{3 \rho_{0}}\right)^{2}+\frac{Q_{0}}{6}\left(\frac{\rho-\rho_{0}}{3 \rho_{0}}\right)^{3}+\mathcal{O}(4)
$$

and the isovector part $S(\rho)$ as

$$
S(\rho)=J_{0}+L_{0}\left(\frac{\rho-\rho_{0}}{3 \rho_{0}}\right)+\frac{K_{\mathrm{sym}, 0}}{2}\left(\frac{\rho-\rho_{0}}{3 \rho_{0}}\right)^{2}+\mathcal{O}(3),
$$

where $J_{0}=S\left(\rho_{0}\right)$ is the symmetry energy coefficient. The incompressibility $K_{0}$, the skewness coefficient $Q_{0}$, the slope $L_{0}$, and the curvature $K_{\mathrm{sym}, 0}$ of the symmetry energy are defined in, e.g., Ref. [5].

The slope of the incompressibility, $M_{0}$, at saturation density, and $K_{\tau, 0}$ are defined as [18]

$$
\begin{aligned}
M_{0} & =Q_{0}+12 K_{0}, \\
K_{\tau, 0} & =K_{\text {sym }, 0}-6 L_{0}-\frac{Q_{0}}{K_{0}} L_{0} .
\end{aligned}
$$

We use a representative set of RMF models, a set of Skyrme-type models, and two microscopic calculations for our correlation study. The RMF models can be classified broadly into two categories: (1) models with nonlinear self and/or mixed interaction terms and constant coupling strengths and (2) models with only linear interaction terms but densitydependent coupling strengths. The type I models used in the present calculations are BSR2, BSR3, BSR6 [19,20], FSU2 [21], GM1 [22], NL3 [23], NL3 $\sigma \rho 4$, NL3 $\sigma \rho 6$ [24], NL3 $\omega \rho 02$ [2], NL3 $\omega \rho 03$ [25], TM1 [26], and TM1-2 [27]. The type II models are DD2 [28], DDH $\delta$ [29], DDH $\delta \operatorname{Mod}$ [7], DDME1 [30], DDME2 [31], and TW [32]. The Skyrme models we use in this work are SKa, SKb [33], SkI2, SkI3, SkI4, SkI5 [34], SkI6 [35], Sly2, Sly9 [36], Sly230a [37], Sly4 [38], SkMP [39], SKOp [40], KDE0V1 [41], SK255, SK272 [42], Rs [43], BSk20, BSk21 [44], BSk22, BSk23, BSk24, BSk25, and BSk26 [45]. The microscopic calculations include the BHF EoS from $[16,46]$, and the APR EoS is taken from $[7,17,47]$. The values of the EoS parameters at saturation density show a wide variation across the models. The symmetric nuclear matter properties for these models are presented in Table I of the Supplemental Material [48]. We shall mainly focus on the correlations between the neutron star radii and the various key parameters of the EoSs: $K_{0}, Q_{0}$, $M_{0}, J_{0}, L_{0}, K_{\text {sym }, 0}$, and $K_{\tau, 0}$, which are evaluated at saturation density.

It was shown in Ref. [14] that nonunified EoSs may introduce a large uncertainty on the determination of low-mass star radii, i.e., $M_{\mathrm{NS}} \lesssim 1.4 M_{\odot}$, mainly if the behaviours of the symmetry energy slope for the EoSs of the inner crust and core are very different. For the RMF models, the EoSs for $\beta$-equilibrated matter are built according to the following procedure. The EoS for the outer crust region is taken from

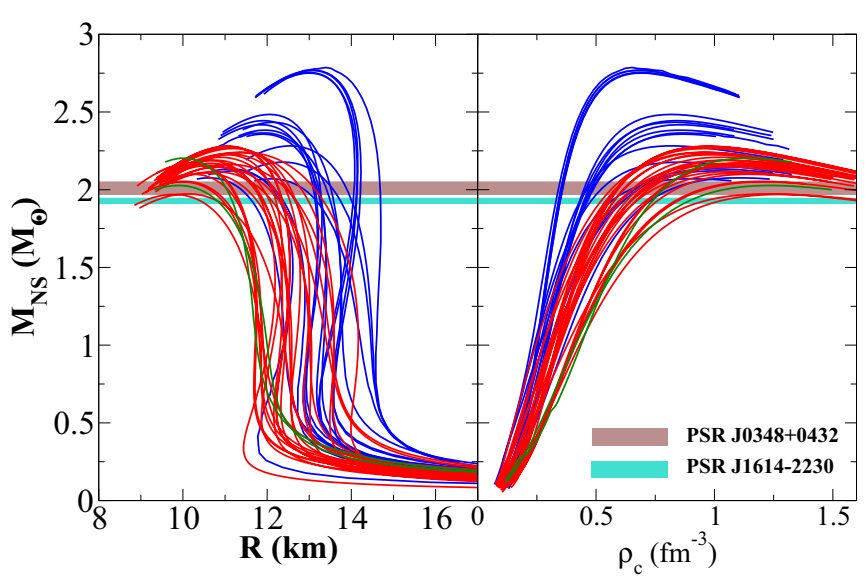

FIG. 1. Neutron star mass in $M_{\odot}$ as a function of the radius in $\mathrm{km}$ (left) and central density in $\mathrm{fm}^{-3}$ (right) for a representative set of RMF (blue) and Skyrme (red) models, and microscopic (green) calculations.

the work of Baym-Pethick-Sutherland [49]. For the inner crust region, we use the EoS including the pasta phases, if they exist, obtained within a Thomas-Fermi calculation [50] up to the crust-core transition density, $\rho_{t}$. At the crust-core transition, the inner crust EoS is matched to the corresponding homogeneous EoS. The fraction of the particles at a given density is determined by imposing $\beta$-equilibrium and charge neutrality. The model used for the outer crust is not the same as the one used for the inner crust and the core regions. However, the use of different EoSs for the outer crust has been shown to barely affect the radius of a star for masses above $1 M_{\odot}$ [14]. For the Skyrme models, the same functional is used for the crust and the core. In the crust, a compressible liquid-drop model (CLDM) and a variational approach, detailed in [14,51], are employed to describe the nuclei. Finally, for the BHF and APR EoSs, the outer and inner crusts are described by the EoSs of [52] and [53], respectively.

The mass $M_{\mathrm{NS}}$ and radius $R$ of static neutron stars are obtained by solving the Tolman-Oppenheimer-Volkoff equations [54], using all of these 44 EoSs. The mass-radius relations are plotted in Fig. 1 (left panel), where the horizontal strips indicate the masses of the two heaviest neutron stars observed so far: PSR J0348+0432 [55] and PSR J1614-2230 [56,57]. For the BSk models, the $M-R$ relations obtained with EoSs based on a simplified CLDM used in this work are close to the ones calculated with a full microscopic model in [58,59]; see the discussion in [14]. To facilitate our discussion, we also display the mass as a function of central density in the right panel of the same figure. The EoSs give rise to different neutron star properties. The spread in the maximum mass is $\sim 0.8 M_{\odot}$, and the spread in the radius of a neutron star with canonical mass $\left(1.4 M_{\odot}\right)$ is $\sim 3.1 \mathrm{~km}$. In Table II of the Supplemental Material [48], we provide the maximum masses together with the radii for different neutron star masses, calculated for all the models used in this study.

The values of the EoS parameters and neutron star radii, obtained for these models, will be used to study the correlations between these quantities. A linear correlation between any two 


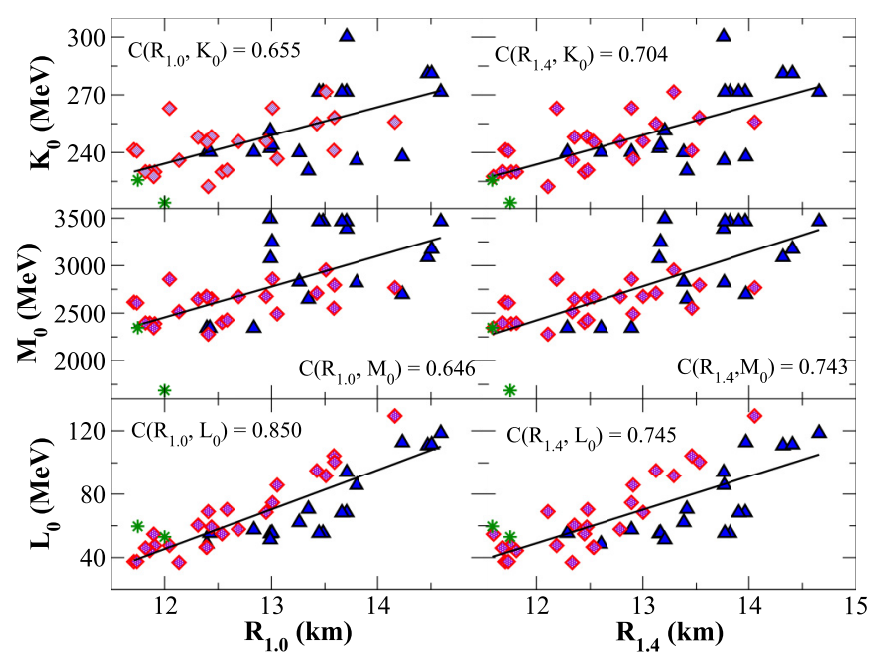

FIG. 2. Radii $R_{1.0}$ (left) and $R_{1.4}$ (right) of $1.0 M_{\odot}$ and $1.4 M_{\odot}$ neutron stars versus the EoS parameters $K_{0}, M_{0}$, and $L_{0}$, obtained using our sets of RMF (blue triangles) and Skyrme (red diamonds) models, together with the BHF and the APR (green stars) calculations.

quantities, $a$ and $b$, can be quantitatively studied by Pearson's correlation coefficient, $C(a, b)$, given by

$$
C(a, b)=\frac{\sigma_{a b}}{\sqrt{\sigma_{a a} \sigma_{b b}}},
$$

with the covariance, $\sigma_{a b}$, written as

$$
\sigma_{a b}=\frac{1}{N_{m}} \sum_{i} a_{i} b_{i}-\left(\frac{1}{N_{m}} \sum_{i} a_{i}\right)\left(\frac{1}{N_{m}} \sum_{i} b_{i}\right),
$$

where the index $i$ runs over the number of models $N_{m}$ [60]. In what follows, $a_{i}$ and $b_{i}$ correspond to the neutron star radius for a fixed mass and an EoS parameter, respectively, obtained for the different models. A correlation coefficient equal to 1 in absolute value indicates a perfect linear relation between the two quantities that are considered.

In Fig. 2, we plot the radii of $1.0 M_{\odot}$ and $1.4 M_{\odot}$ stars, $R_{1.0}$ and $R_{1.4}$, versus some of these EoS parameters for our representative sets of RMF (blue triangles) and Skyrme (red diamonds) models, together with the BHF and the APR (green stars) calculations. The solid lines in the figures are obtained by linear regression and the correlation coefficients are indicated for each case considered. The correlations between the neutron star radius and the isoscalar parameters $K_{0}$ and $M_{0}$ increase with the increase of the neutron star mass; however, they are not significantly strong to make a meaningful prediction. The $R_{1.4}-L_{0}$ correlation is weaker than the $R_{1.0}-L_{0}$ correlation, which is opposite to the trend observed for the cases of $K_{0}$ and $M_{0}$. In Table I, we list all the correlation coefficients between the EoS parameters $K_{0}, M_{0}, Q_{0}, J_{0}, L_{0}, K_{\text {sym }, 0}$, and $K_{\tau, 0}$, and the radii of neutron stars with different masses.

The study of the correlations clearly indicates that the radius of low-mass neutron stars is more sensitive to the isovector EoS parameters $\left(J_{0}\right.$ and $\left.L_{0}\right)$, but, as the mass of the neutron star increases, the sensitivity to the isoscalar parameters $\left(K_{0}\right.$ and $M_{0}$ ) tend to dominate. A similar conclusion was drawn in Ref. [14], where the behavior of the radius of stars with
TABLE I. Correlation coefficients between the neutron star radii and the different EoS parameters obtained for a representative set of RMF, Skyrme, and microscopic calculations. The EoS parameters are the nuclear matter incompressibility coefficient $K_{0}$, its skewness $Q_{0}$ and slope $M_{0}$, the symmetry energy coefficient $J_{0}$, its slope $L_{0}$ and curvature $K_{\text {sym }, 0}$, and the parameter $K_{\tau, 0}$, calculated at the saturation density. $R_{x}$ denotes the neutron star radius for a given mass $x$ in units of $M_{\odot}$.

\begin{tabular}{cccccccc}
\hline \hline & $K_{0}$ & $Q_{0}$ & $M_{0}$ & $J_{0}$ & $L_{0}$ & $K_{\text {sym }, 0}$ & $K_{\tau, 0}$ \\
\hline$R_{0.6}$ & 0.565 & 0.383 & 0.548 & 0.815 & 0.887 & 0.581 & -0.809 \\
$R_{0.8}$ & 0.617 & 0.416 & 0.597 & 0.743 & 0.881 & 0.658 & -0.775 \\
$R_{1.0}$ & 0.655 & 0.461 & 0.646 & 0.680 & 0.850 & 0.698 & -0.743 \\
$R_{1.2}$ & 0.684 & 0.514 & 0.695 & 0.621 & 0.803 & 0.714 & -0.716 \\
$R_{1.4}$ & 0.704 & 0.571 & 0.743 & 0.562 & 0.745 & 0.711 & -0.689 \\
$R_{1.6}$ & 0.718 & 0.628 & 0.787 & 0.502 & 0.674 & 0.691 & -0.661 \\
$R_{1.8}$ & 0.725 & 0.686 & 0.828 & 0.438 & 0.590 & 0.653 & -0.630 \\
\hline \hline
\end{tabular}

mass $M_{\mathrm{NS}}=1.0,1.4,1.8 M_{\odot}$ for 33 models, including $9 \mathrm{RMF}$ models and 24 Skyrme forces, were plotted as a function of $K_{0}$ and $L_{0}$. The correlation coefficient $C\left(R_{1.0}, L_{0}\right)$ was 0.87 , while $C\left(R_{1.8}, L_{0}\right)$ was 0.64 . The value of $C\left(R_{1.0}, K_{0}\right)$ was 0.63 , whereas the values of $C\left(R_{1.4}, K_{0}\right)$ and $C\left(R_{1.8}, K_{0}\right)$ were found to be $\sim 0.66$. These values are in good agreement with the values we find in this work, especially for the correlation coefficients of the low-mass neutron star radii.

Next we look into the correlations of neutron star radii with selected combinations of isoscalar and isovector EoS parameters. In Fig. 3, we plot the neutron star radii for $M_{\mathrm{NS}}=1.0 M_{\odot}$ (left) and $1.4 M_{\odot}$ (right) as a function of the linear combinations, $K_{0}+\alpha L_{0}$ (top), and $M_{0}+\beta L_{0}$ (bottom). We can see that the neutron star radii are better correlated with these combinations, than with the each of the parameter separately, as seen in Fig. 2. Further, the strongest correlations occur between the neutron star radii and the linear combination $M_{0}+\beta L_{0}$. In Table II, we list again all the
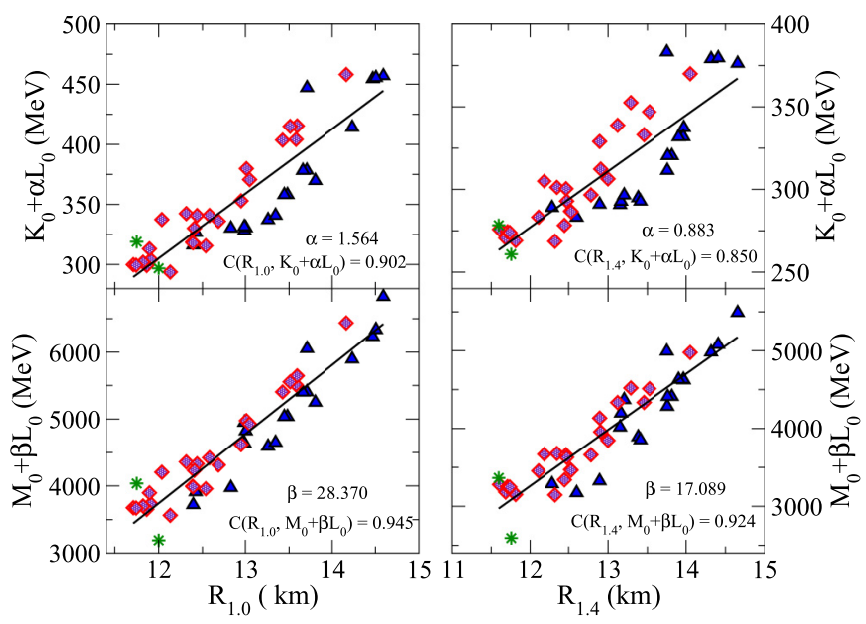

FIG. 3. Neutron star radii $R_{1.0}$ (left) and $R_{1.4}$ (right) versus the linear correlations $K_{0}+\alpha L_{0}$ (top) and $M_{0}+\beta L_{0}$ (bottom), using a set of RMF (blue triangles), Skyrme (red diamonds), and BHF+APR (green stars) calculations. 
TABLE II. The correlation coefficients of neutron star radii with $K_{0}+\alpha L_{0}$ and $M_{0}+\beta L_{0}$, along with the values of $\alpha$ and $\beta$.

\begin{tabular}{cccccc}
\hline \hline & \multicolumn{2}{c}{$K_{0}+\alpha L_{0}$} & & \multicolumn{2}{c}{$M_{0}+\beta L_{0}$} \\
\cline { 2 - 3 } \cline { 5 - 6 } & $\alpha$ & Corr. coeff. & & $\beta$ & Corr. coeff. \\
\hline$R_{0.6}$ & 2.970 & 0.905 & & 43.115 & 0.936 \\
$R_{0.8}$ & 2.111 & 0.914 & & 35.575 & 0.949 \\
$R_{1.0}$ & 1.564 & 0.902 & & 28.370 & 0.945 \\
$R_{1.2}$ & 1.177 & 0.879 & & 22.189 & 0.935 \\
$R_{1.4}$ & 0.883 & 0.850 & & 17.089 & 0.924 \\
$R_{1.6}$ & 0.643 & 0.817 & & 12.781 & 0.913 \\
$R_{1.8}$ & 0.432 & 0.782 & 8.970 & 0.903 \\
\hline \hline
\end{tabular}

correlation coefficients of neutron star radii with $K_{0}+\alpha L_{0}$ and $M_{0}+\beta L_{0}$, for different neutron star masses. The values of $\alpha$ and $\beta$, also listed, are obtained in such a way that the correlations of these quantities with the neutron star radii are maximum.

In the top panel of Fig. 4, we show the variation of the correlation coefficients of neutron star radii with $K_{0}, L_{0}$, and $K_{0}+\alpha L_{0}$, as a function of the mass of the star. The correlation of neutron star radii with $K_{0}+\alpha L_{0}$ is better than those with $K_{0}$ and $L_{0}$ individually. However, for $M_{\mathrm{NS}} \gtrsim 1.0 M_{\odot}$, the correlations of neutron star radii decrease gradually with the increase of the neutron star mass, even considering $K_{0}+\alpha L_{0}$. In the bottom panel of Fig. 4, we repeat the same exercise for $M_{0}, L_{0}$, and $M_{0}+\beta L_{0}$. Again, contrary to the individual parameters $M_{0}$ and $L_{0}$, the neutron star radii are strongly correlated with $M_{0}+\beta L_{0}$ over a wide range of neutron star masses $\left(0.6-1.8 M_{\odot}\right)$.

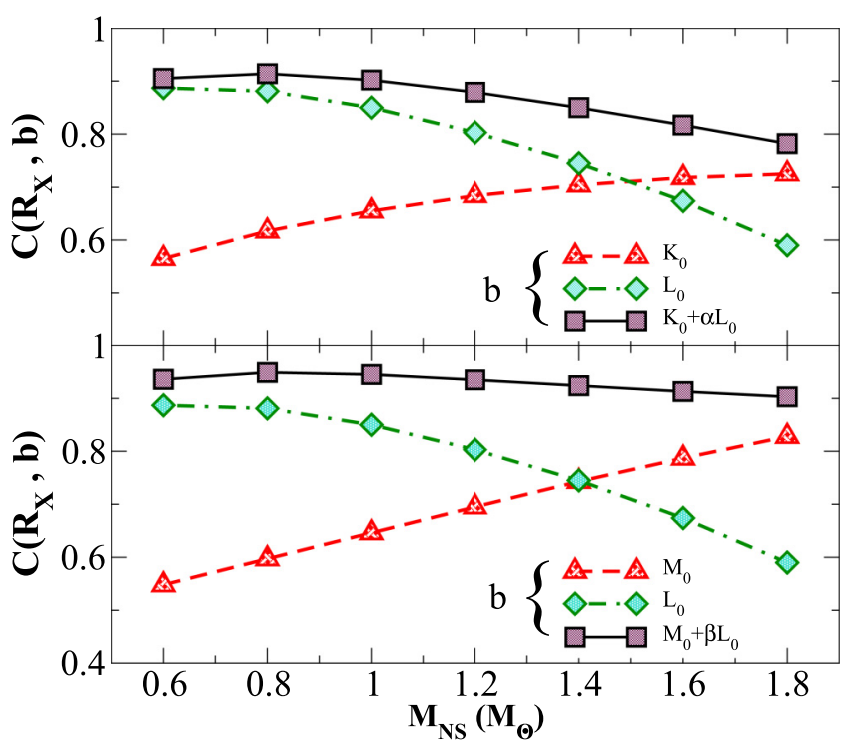

FIG. 4. Correlation coefficients between the neutron star radii and several EoS parameters as a function of the neutron star mass. The EoS parameters " $b$ " denote $K_{0}, L_{0}$, and the linear combination $K_{0}+\alpha L_{0}$ in the top panel, and $M_{0}, L_{0}$, and $M_{0}+\beta L_{0}$ in the bottom panel.
In order to interpret the correlations obtained, we consider the dependence of the pressure on the isoscalar coefficients, $K_{0}, M_{0}, Q_{0}$, and on the slope of the symmetry energy, $L_{0}$. Taking the expansions given in (2) and (3), the pressure is given by

$$
P=\frac{\rho_{0} x^{2}}{3}\left[\frac{K_{0}}{3}(x-1)+\frac{Q_{0}}{18}(x-1)^{2}+L_{0} \delta^{2}\right],
$$

with $x=\rho / \rho_{0}$, or expressing $Q_{0}$ in terms of $M_{0}$ and $K_{0}$, by

$$
P=\frac{\rho_{0} x^{2}}{3}\left[K_{0}(x-1)\left(1-\frac{2 x}{3}\right)+\frac{M_{0}}{18}(x-1)^{2}+L_{0} \delta^{2}\right]
$$

These two equations and the empirical relation $R \propto P^{1 / 4}$, identified in Ref. [3,61], where $R$ is the star radius and $P$ the pressure, calculated for some fiducial density, $\sim 1-2 \rho_{0}$, allow an interpretation of the above correlations of $R$ with $K_{0}+\alpha L_{0}$ and $M_{0}+\beta L_{0}$.

In the following, we present some arguments that explain the correlations: (a) If $\rho=\rho_{0}$, only the $L_{0}$ term survives and this may explain why the radius of low-mass neutron stars is well correlated with $L_{0}$. (b) From Eq. (9), it is seen that, for $\rho=1.5 \rho_{0}$, the pressure depends only on $M_{0}$ and $L_{0}$. This behavior explains why the correlation of $M_{0}$ with the star radius shown in Fig. 2 is better for $R_{1.4}$ than for $R_{1.0}$, and also why the correlation of $R$ with $M_{0}+\beta L_{0}$ is so strong. (c) The contribution of the $K_{0}$ term in (8) is more important than the $Q_{0}$ term for $x-1<1$, which explains the correlation of $R$ with $K_{0}+\alpha L_{0}$; Finally (d) the asymmetry parameter $\delta$ monotonically decreases from its maximum value $\sim 0.95$, obtained at densities of the order of $\rho_{0} / 2$ to $0.65<\delta<0.85$ at $2 \rho_{0}$. If the term in $K_{0}$ is neglected in Eq. (9), the pressure satisfies $P \propto M_{0}+\frac{18 \delta^{2}}{(x-1)^{2}} L_{0}=M_{0}+\beta^{\prime} L_{0}$. Taking for $\beta^{\prime}$ the upper and lower values of $\beta$ in Table II, we get, respectively, $x=1.45$ and $x=1.99$, above and just below the value $x=$ 1.5 , when the relation is exact. Therefore, it seems the relation is being applied within the valid range of density. On the other hand, from Eq. (8) and neglecting the term in $Q_{0}$, the relation $P \propto K_{0}+\frac{3 \delta^{2}}{(x-1)} L_{0}=K_{0}+\alpha^{\prime} L_{0}$, is obtained. We now take for $\alpha^{\prime}$ the upper and lower values of $\alpha$ from Table II, and we get, respectively, $x=1.49$ and $x=4.40$. The last value is already out of the validity of the approximation, and even for $x=2$ this approximation is not very good. This might be the plausible reason that only the low-mass neutron star radii are strongly correlated with $K_{0}+\alpha L_{0}$.

Knowledge of the slopes of nuclear matter incompressibility and symmetry energy at saturation density can constrain the neutron star radii, as these radii are strongly correlated with $M_{0}+\beta L_{0}$. An overall variation in $L_{0}=20-80 \mathrm{MeV}$ is obtained from the analysis of the giant dipole resonance of ${ }^{208} \mathrm{~Pb}[63,64]$, the giant quadrupole resonance in ${ }^{208} \mathrm{~Pb}[65]$, the pigmy dipole resonance in ${ }^{68} \mathrm{Ni}$ and ${ }^{132} \mathrm{Sn}$ [66], and nuclear ground state properties, using the standard Skyrme Hartree-Fock approach [67]. A fit of the EoS for asymmetric nuclear matter, or pure neutron matter $[38,68]$, or the binding energies of large number of deformed nuclei $[69,70]$ within different mean field models, constrains the value of $L_{0}$ in 


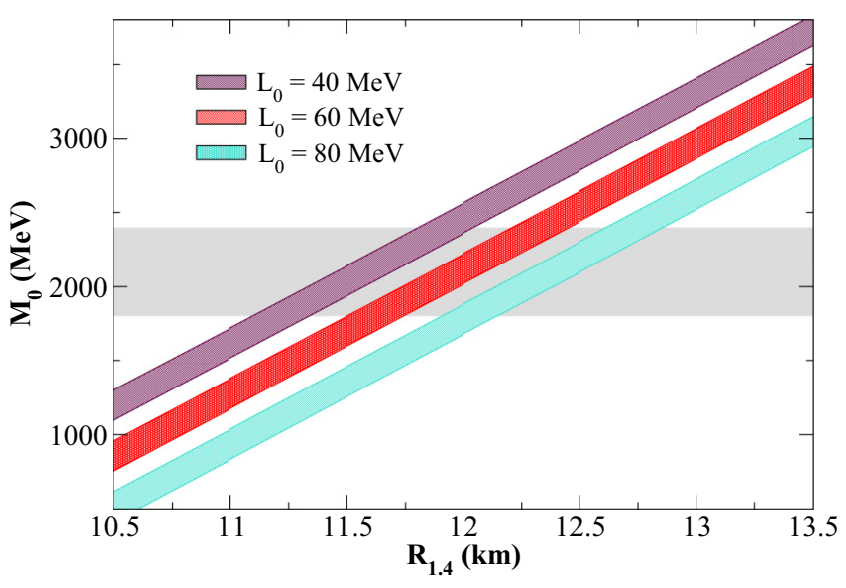

FIG. 5. $M_{0}$ as a function of $R_{1.4}$ for $L_{0}=40,60$, and $80 \mathrm{MeV}$, as obtained from the multiple linear regression. The gray shaded region indicates the constraint on $M_{0}$ derived in Ref. [62].

the range of $40-70 \mathrm{MeV}$. The combined results of nuclear structure and heavy ion collisions lead to the central value of $L_{0}=70 \mathrm{MeV}$ [71]. We adopt $L_{0}=40-80 \mathrm{MeV}$, which has a good overlap with these investigations. The value of $M_{0}=1800-2400 \mathrm{MeV}$ [62] at the saturation density seems to be consistent with its value at $\rho=0.1 \mathrm{fm}^{-3}$, deduced from the energies of the isoscalar giant monopole resonance in the ${ }^{132} \mathrm{Sn}$ and ${ }^{208} \mathrm{~Pb}$ nuclei [72,73]. In Fig. 5, we plot the incompressibility slope parameter $M_{0}$ as a function of $R_{1.4}$, for different values of the symmetry energy slope $L_{0}=40,60$, and $80 \mathrm{MeV}$. The gray shaded region corresponds to the constraint on $M_{0}$ as obtained in Ref. [62]. These values of $M_{0}$ and $L_{0}$ suggest that $R_{1.4}$ should be in the range of $11.09-12.86 \mathrm{~km}$, which is consistent with the results of the Ref. [74]. Let us remark that if we had only taken the RMF models, the above correlations would have been slightly stronger, as expected, because all models in the study would have had a similar underlying framework, and larger radii would have been obtained for a $1.4 M_{\odot}$ star, namely $R_{1.4}$ would have come out in the range of $11.82-13.25 \mathrm{~km}$. Indeed Fig. 1 shows that, on average, RMF models lead to larger radii than the other types of calculations.

In conclusion, we have studied the possible existing correlations between neutron star radii at different masses and the nuclear coefficients of the nuclear matter EoSs, calculated at the saturation density. The neutron star radii are obtained using unified EoSs, fully for the Skyrme models and partially for inner-crust-core EoSs for the RMF models, except for the two microscopic EoSs. All EoSs are consistent with the existence of $2 M_{\odot}$ neutron stars. The radii of the low-mass neutron stars are better correlated with the symmetry energy coefficient $J_{0}$ and its slope $L_{0}$. As the neutron star mass increases, the correlations of the radii with the nuclear matter incompressibility $K_{0}$ and its slope $M_{0}$ grow stronger. Our investigation reveals that the neutron star radii are better correlated with the linear combinations $K_{0}+\alpha L_{0}$ and $M_{0}+\beta L_{0}$ than with the individual EoS parameters. In particular, noticeable improvement is seen in the correlations of the radii with these linear combinations, for $1.4 M_{\odot}$ neutron stars. The correlations of the radii with $M_{0}+\beta L_{0}$ are stronger, and almost independent of the neutron star mass, in the range $0.6-1.8 M_{\odot}$. A plausible interpretation for the existence of such correlations is traced back to the correlations between the pressure and similar linear combinations of the EoS parameters in the relevant density range. The values of $M_{0}$ and $L_{0}$, as currently deduced from finite nuclei data, constrain $R_{1.4}$ in the range $11.09-12.86 \mathrm{~km}$.

H.P. is supported by FCT under Project No. SFRH/BPD/95566/2013. Partial support comes from FCT under project reference UID/FIS/04564/2016 and "NewCompstar", COST Action MP1304. The work of M.F. has been partially supported by the NCN (Poland) Grant No. 2014/13/B/ST9/02621.
[1] N. K. Glendenning, Phys. Rev. Lett. 57, 1120 (1986).

[2] C. J. Horowitz and J. Piekarewicz, Phys. Rev. Lett. 86, 5647 (2001).

[3] J. M. Lattimer and M. Prakesh, Phys. Rep. 442, 109 (2007).

[4] J. Xu, L.-W. Chen, B.-A. Li, and H.-R. Ma, Astrophys. J. 697, 1549 (2009).

[5] I. Vidaña, C. Providência, A. Polls, and A. Rios, Phys. Rev. C 80, 045806 (2009).

[6] C. Ducoin, J. Margueron, and C. Providência, Europhys. Lett. 91, 32001 (2010).

[7] C. Ducoin, J. Margueron, C. Providência, and I. Vidaña, Phys. Rev. C 83, 045810 (2011).

[8] R. Cavagnoli, D. P. Menezes, and C. Providência, Phys. Rev. C 84, 065810 (2011).

[9] S. Gandolfi, J. Carlson, and S. Reddy, Phys. Rev. C 85, 032801 (2012).
[10] F. J. Fattoyev and J. Piekarewicz, Phys. Rev. C 86, 015802 (2012).

[11] W. G. Newton, M. Gearheart, and B.-A. Li, Astrophys. J. Suppl. 204, 9 (2013).

[12] F. J. Fattoyev, W. G. Newton, and B.-A. Li, Phys. Rev. C 90, 022801(R) (2014).

[13] H. Sotani, K. Iida, and K. Oyamatsu, Phys. Rev. C 91, 015805 (2015).

[14] M. Fortin, C. Providência, A. R. Raduta, F. Gulminelli, J. L. Zdunik, P. Haensel, and M. Bejger, Phys. Rev. C 94, 035804 (2016).

[15] M. Dutra, O. Lourenço, and D. P. Menezes, Phys. Rev. C 93, 025806 (2016).

[16] G. Taranto, M. Baldo, and G. F. Burgio, Phys. Rev. C 87, 045803 (2013).

[17] A. Akmal, V. R. Pandharipande, and D. G. Ravenhall, Phys. Rev. C 58, 1804 (1998). 
[18] N. Alam, B. K. Agrawal, J. N. De, S. K. Samaddar, and G. Colò, Phys. Rev. C 90, 054317 (2014).

[19] S. K. Dhiman, R. Kumar, and B. K. Agrawal, Phys. Rev. C 76, 045801 (2007).

[20] B. K. Agrawal, Phys. Rev. C 81, 034323 (2010).

[21] W.-C. Chen and J. Piekarewicz, Phys. Rev. C 90, 044305 (2014).

[22] N. K. Glendenning and S. A. Moszkowski, Phys. Rev. Lett. 67, 2414 (1991).

[23] G. A. Lalazissis, J. König, and P. Ring, Phys. Rev. C 55, 540 (1997).

[24] H. Pais and C. Providência, Phys. Rev. C 94, 015808 (2016).

[25] J. Carriere, C. Horowitz, and J. Piekarewicz, Astrophys. J. 593, 463 (2003).

[26] Y. Sugahara and H. Toki, Nucl. Phys. A 579, 557 (1994).

[27] C. Providência and A. Rabhi, Phys. Rev. C 87, 055801 (2013).

[28] S. Typel, G. Ropke, T. Klahn, D. Blaschke, and H. H. Wolter, Phys. Rev. C 81, 015803 (2010).

[29] T. Gaitanos, M. Di Toro, S. Typel, V. Baran, C. Fuchs, V. Greco, and H. H. Wolter, Nucl. Phys. A 732, 24 (2004).

[30] T. Nikšić, D. Vretenar, P. Finelli, and P. Ring, Phys. Rev. C 66, 024306 (2002).

[31] G. A. Lalazissis, T. Nikšić, D. Vretenar, and P. Ring, Phys. Rev. C 71, 024312 (2005).

[32] S. Typel and H. H. Wolter, Nucl. Phys. A 656, 331 (1999).

[33] H. Kohler, Nucl. Phys. A 258, 301 (1976).

[34] P.-G. Reinhard and H. Flocard, Nucl. Phys. A 584, 467 (1995).

[35] W. Nazarewicz, J. Dobaczewski, T. R. Werner, J. A. Maruhn, P.-G. Reinhard, K. Rutz, C. R. Chinn, A. S. Umar, and M. R. Strayer, Phys. Rev. C 53, 740 (1996).

[36] E. Chabanat, Ph.D. thesis, University Claude Bernard Lyon-1, Lyon, France, 1995 (unpublished).

[37] E. Chabanat, P. Bonche, P. Haensel, J. Meyer, and R. Schaeffer, Nucl. Phys. A 627, 710 (1997).

[38] E. Chabanat, P. Bonche, P. Haensel, J. Meyer, and R. Schaeffer, Nucl. Phys. A 635, 231 (1998).

[39] L. Bennour, P.-H. Heenen, P. Bonche, J. Dobaczewski, and H. Flocard, Phys. Rev. C 40, 2834 (1989).

[40] P. G. Reinhard, Nucl. Phys. A 649, 305 (1999).

[41] B. K. Agrawal, S. Shlomo, and V. K. Au, Phys. Rev. C 72 014310 (2005).

[42] B. K. Agrawal, S. Shlomo, and V. Kim Au, Phys. Rev. C 68, 031304 (2003).

[43] J. Friedrich and P.-G. Reinhard, Phys. Rev. C 33, 335 (1986).

[44] S. Goriely, N. Chamel, and J. M. Pearson, Phys. Rev. C 82, 035804 (2010).

[45] S. Goriely, N. Chamel, and J. M. Pearson, Phys. Rev. C 88, 024308 (2013).

[46] D. Davesne, A. Pastore, and J. Navarro, Astron. Astrophys. 585, A83 (2016).

[47] A. W. Steiner, M. Prakash, J. M. Lattimer, and P. J. Ellis, Phys. Rep. 411, 325 (2005).
[48] See Supplemental Material at http://link.aps.org/supplemental/ 10.1103/PhysRevC.94.052801 for nuclear matter characteristics of various models and resulting properties of neutron stars.

[49] G. Baym, C. Pethick, and P. Sutherland, Astrophys. J. 170, 299 (1971).

[50] F. Grill, H. Pais, C. Providência, I. Vidaña, and S. S. Avancini, Phys. Rev. C 90, 045803 (2014).

[51] F. Gulminelli and A. R. Raduta, Phys. Rev. C 92, 055803 (2015).

[52] P. Haensel, J. L. Zdunik, and J. Dobaczewski, Astron. Astrophys. 222, 353 (1989).

[53] J. W. Negele and D. Vautherin, Nucl. Phys. A 207, 298 (1973).

[54] S. Weinberg, Gravitation and Cosmology (Wiley, New York, 1972).

[55] J. Antoniadis et al., Science 340, 448 (2013).

[56] P. B. Demorest, T. Pennucci, S. M. Ransom, M. S. E. Roberts, and J. W. T. Hessels, Nature 467, 1081 (2010).

[57] E. Fonseca et al., arXiv:1603.00545.

[58] A. F. Fantina, N. Chamel, J. M. Pearson, and S. Goriely, EPJ Web Conf. 66, 07005 (2014).

[59] J. M. Pearson, N. Chamel, A. F. Fantina, and S. Goriely, Eur. Phys. J. A 50, 43 (2014).

[60] S. Brandt, Statistical and Computational Methods in Data Analysis, 3rd ed. (Springer, New York, 1997).

[61] J. M. Lattimer and M. Prakash, Astrophys. J. 550, 426 (2001).

[62] J. N. De, S. K. Samaddar, and B. K. Agrawal, Phys. Rev. C 92, 014304 (2015).

[63] L. Trippa, G. Colò, and E. Vigezzi, Phys. Rev. C 77, 061304(R) (2008)

[64] X. Roca-Maza, M. Brenna, G. Colò, M. Centelles, X. Viñas, B. K. Agrawal, N. Paar, D. Vretenar, and J. Piekarewicz, Phys. Rev. C 88, 024316 (2013).

[65] X. Roca-Maza, M. Brenna, B. K. Agrawal, P. F. Bortignon, G. Colò, L.-G. Cao, N. Paar, and D. Vretenar, Phys. Rev. C 87, 034301 (2013).

[66] A. Carbone, G. Colò, A. Bracco, L.-G. Cao, P. F. Bortignon, F. Camera, and O. Wieland, Phys. Rev. C 81, 041301(R) (2010).

[67] L.-W. Chen, Phys. Rev. C 83, 044308 (2011).

[68] X. Roca-Maza, X. Vinas, M. Centelles, P. Ring, and P. Schuck, Phys. Rev. C 84, 054309 (2011).

[69] T. Nikšić, D. Vretenar, and P. Ring, Phys. Rev. C 78, 034318 (2008).

[70] P. W. Zhao, Z. P. Li, J. M. Yao, and J. Meng, Phys. Rev. C 82, 054319 (2010).

[71] M. B. Tsang, J. R. Stone, F. Camera, P. Danielewicz, S. Gandolfi, K. Hebeler, C. J. Horowitz, J. Lee, W. G. Lynch, Z. Kohley, R. Lemmon, P. Möller, T. Murakami, S. Riordan, X. Roca-Maza, F. Sammarruca, A. W. Steiner, I. Vidaña, and S. J. Yennello, Phys. Rev. C 86, 015803 (2012).

[72] E. Khan, J. Margueron, and I. Vidaña, Phys. Rev. Lett. 109, 092501 (2012).

[73] E. Khan and J. Margueron, Phys. Rev. C 88, 034319 (2013).

[74] A. W. Steiner, J. M. Lattimer, and E. F. Brown, Eur. Phys. J. A 52, 18 (2016). 